\title{
Das Geschäftszentrum von St. Gallen
}

Im Laufe der vergangenen Jahre hat das Stadtgebiet von St. Gallen den Lehrveranstaltungen der HSG in Wirtschaftsgeographie als Studien- und Übungsobjekt mehrfach gedient1). Unter verschiedenen Fragen der Stadtstruktur fand vor allem jene der City-Gliederung und City-Abgrenzung eine intensive Bearbeitung. Sie wurde abgerundet durch eine Diplomarbeit2), in der neben der Ausstattung mit städtischen Subzentren die räumliche Ausdehnung des Geschäftszentrums zu erfassen war.

Auf der Basis dieser Aufnahmen und Studien wurde an der Forschungsstelle für Wirtschaftsgeographie und Raumplanung die vorliegende Information ausgearbeitet.

\section{St. Gallen als «Zentraler Ort»}

Stichprobenerhebungen hatten gezeigtl), daß eine große Anzahl von Geschäften in der Altstadt eine Sortimentsstruktur aufweisen, welche nach $\mathrm{CAROL}^{3}$ ) für die Existenz eines Central-Business-Districts symptomatisch sind. Die Einzugsgebiete ausgewählter Branchen im Geschäftszentrum der Altstadt reichen bis in den Raum Sargans, über Wil hinaus und bis in den äußeren Thurgau; die Basisbevölkerung St. Gallens als Zentralem Ort der Stufe «Stadt» bis «Großstadt» umfaßt rund 400000 Einwohner. Die Voraussetzung für eine Citybildung mit einem in das weitere Umland der Stadt ausstrahlenden Bedeutungsüberschuß erscheint damit als gegeben, auch wenn Zentrale Dienste hoher Ordnung (Stadttheater, Hochschule, medizinische Kliniken u. a.) nur teilweise vertreten sind.

\section{Methoden der City-Abgrenzung}

Unter City oder dem höchstwertigen Geschäftsgebiet verstehen wir «einen zentral gelegenen Stadtteil, in dem die zentralörtlichen Funktionen der höchsten Stufe der Bedienung in einzigartiger Konzentration vereinigt sind»4). Die City umfaßt sowohl das Einzelhandelszentrum als auch die übrigen zentralsten Funktionen. Sie stellt innerhalb der zugehörigen Stadt immer den Raum höchster Aktivitäten dar.

KANT unterscheidet zwei fundamentale Kategorien von Methoden für die räumliche Abgrenzung, Kenn- zeichnung und Untergliederung städtischer Kerngebiete (= städtische Nukleonik):

- die Abgrenzung aufgrund des Raumgefüges der einzelnen Merkmalsträger oder Indikatoren,

- die Abgrenzung nach mehreren Bestimmungsmerkmalen oder die synthetische Methodes).

Im Rahmen der vorliegenden geographischen Bestandesaufnahme erfolgt die Abgrenzung der St. Galler City sowohl unter dem physiognomischen wie auch funktionalen Gesichtspunkt. Beide Betrachtungsweisen sind der erstgenannten Kategorie der Indikatoren-Methoden zugeordnet.

\subsection{Physiognomisch-verbale Betrachtungsweise}

Nach KANT ist die physiognomische Betrachtungsweise «eine auf das äußere, formale Erscheinungsbild bzw. auf die bauliche Gestaltung gerichtete Gliederung»s). Die intuitive bzw. optisch-wahrnehmbare Erfassung formaler City-Elemente steht bei der Identifizierung des Stadtkerns im Vordergrund. Die visuelle Beobachtung der äußeren Erscheinung in Grund- und Aufriß kann die historische Entwicklung, das Stadtwachstum und auch funktionale Aspekte der Stadtstruktur ausdrücken.

Die auf die physiognomisch sichtbaren Tatbestände gerichtete Beobachtung genügt jedoch dem wissenschaftlichen Postulat der Nachvollziehbarkeit kaum und ist damit auch kein zweckmäßiges Verfahren zur City-Abgrenzung.

\subsection{Funktional-quantitative Betrachtungsweise}

Die City als städtisches Teilgebiet ist außer durch bestimmte Grund- und Aufrißformen auch durch gemeinsame funktionale Merkmale geprägt und hebt sich damit von benachbarten Stadtteilen ab. Im folgenden werden die funktionalen Aspekte der City aufgrund verschiedener Ansatzpunkte näher untersucht.

Prof. Dr. E. Bugmann, Dr. M. Boesch, cand. oec. J.-P. Jetzer, Forschungsstelle für Wirtschaftsgeographie und Raumplanung an der Hochschule St. Gallen, Guisan-Straße 70, 9010 St. Gallen 


\subsubsection{Das «basic/non basic»-Konzept}

Das «basic/non basic»-Konzept geht von der Reichweite der Funktionen aus und gliedert diese nach ihrem Bedeutungsgehalt für die Stadt in zwei Komponenten7). Den ersten Ansatz zum «basic/non basic»Konzept finden wir bei SOMBART8). Er unterscheidet zwischen lokalen (bedienenden) und überregionalen oder primären Funktionen. Dieses Konzept wurde weiterentwickelt und von HOYT in seiner heutigen Form wiedergegeben9).

Die wirtschaftliche Tätigkeit gliedert sich demzufolge in zwei Komponenten, nämlich:

- in jene, die den Lokalbedarf befriedigt und

- in jene, die eine überregionale Nachfrage zum Gegenstand hat.

Letztere umfaßt Funktionen, die auf Fernwirkungen beruhen. Diese weitreichenden Güter und Dienste sind für das Städtewachstum entscheidend, weshalb sie von SOMBART auch als städtebildende Funktionen (basic oder city forming) bezeichnet worden sind. Die lokalen Funktionen, welche der Eigenversorgung einer Stadtbevölkerung dienen, sind in der Terminologie HOYTs «non basic» oder «city serving».

Für viele «basic»-Funktionen ist die Standortgunst innerhalb der City größer als außerhalb. Das spezialisierte Einzelhandelsangebot und die Dienstleistungen höherer Ordnung entfalten sich insbesondere im städtischen Kerngebiet. Die zentripetale Tendenz der Funktionen des Tertiärsektors ist auf die Kontakthäufigkeit dieser Branchen, verbunden mit der Anforderung an die Erreichbarkeit, zurückzuführen.

\subsubsection{Abstufung der Nutzungsintensität}

Die von MURPHY und VANCE entwickelte Methode zur Bestimmung des CBD (Central Business District) benützt die innenstädtische Flächennutzung als Kriterium der Geschäftsgebietsabgrenzung und -differenzierung10).

In Anlehnung an diese Methode haben wir versucht, in der St. Galler Innenstadt eine Geschäftszone auszuscheiden. Dabei wurde die Systematik der Nutzungsgruppen (NG) nach woLF zugrunde gelegt11). Als $\mathrm{Maß}$ der Nutzungsintensität dient der CBI-Index (central business intensity index, CBII), der wie folgt definiert ist:

$$
\mathrm{CBII}=\frac{\mathrm{CB} \text { Nutzflächen } \cdot 100}{\text { Geschoßflächen }}
$$

Als Bezugsfläche wird jedoch nicht der für amerikanische Verhältnisse typische Straßenblock, sondern das einzelne Gebäude herangezogen. Aufgrund der gebäudeweise vorliegenden Indexwerte konnte eine Isoliniendarstellung der Nutzungsintensität in der St. Galler Innenstadt entwickelt werden12).

Der Hauptteil der Altstadt und das Gebiet bis zum Bahnhof liegen innerhalb der 60\%-Isolinie. Dies bedeutet, daß bei der meist fünfgeschossigen Bauweise in der St. Galler Innenstadt jeweils mindestens drei der fünf Geschosse den analysierten Zentralen Nutzungen dienen. Diese intensiv genutzten Gebiete können damit als das eigentliche Geschäftszentrum St. Gallens bezeichnet werden.

\subsubsection{Die Kern-Mantel-Struktur der St. Galler Innenstadt}

Aufgrund der Abhängigkeit zwischen Grundstückpreisen und Funktionsverteilung entwickelten HORWOOD und BOYCE ein Kern-Mantel-Modell der Geschäftszentren13). Sie unterscheiden einen inneren Kernbereich (hard core) hoher Bodenwerte und intensiver Vertikalnutzung und einen sich anschließenden Ring (frame oder fringe) geringerer Bodenwerte bei gleichzeitig schwächerer Vertikalerstreckung.

Analog der Ausscheidung von Kern und Mantel in Kapstadt, wie dies DAVIES vorgenommen hat, sollen in der St. Galler Innenstadt ein Geschäftskern hoher Nutzungsintensität und eine sich anlehnende Mantelzone geringerer Geschäftsdichte herausgeschält werden14). Dazu werden die Indexwerte wie folgt festgelegt:15)

\section{Kernbereich: $\quad \mathrm{CBII} \geqslant 80 \%$ \\ Mantelzone: $80 \%>\mathrm{CBII} \geqslant 40 \%$}

Damit wird der empirisch ermittelte Schwellenwert von $60 \%$ für das Geschäftszentrum (CBD) berücksichtigt.

Sehr deutlich läßt sich die doppelte Ausprägung der Schwerpunkte des Geschäftslebens im Bahnhofsviertel («Citybereich»16)) und in der Altstadt herauskristallisieren. Die Mantelzonen lehnen sich unmittelbar an die Kernbereiche an. Bezeichnend ist ihre schwache Ausprägung im Bahnhofquartier, womit das dort lokalisierte Verwaltungszentrum der Stadt St. Gallen17) seinen deutlichen Niederschlag findet (vgl. Abb. 1). 
Abbildung 1: Kern- und Mantelbereiche des St. Galler Geschäftszentrums

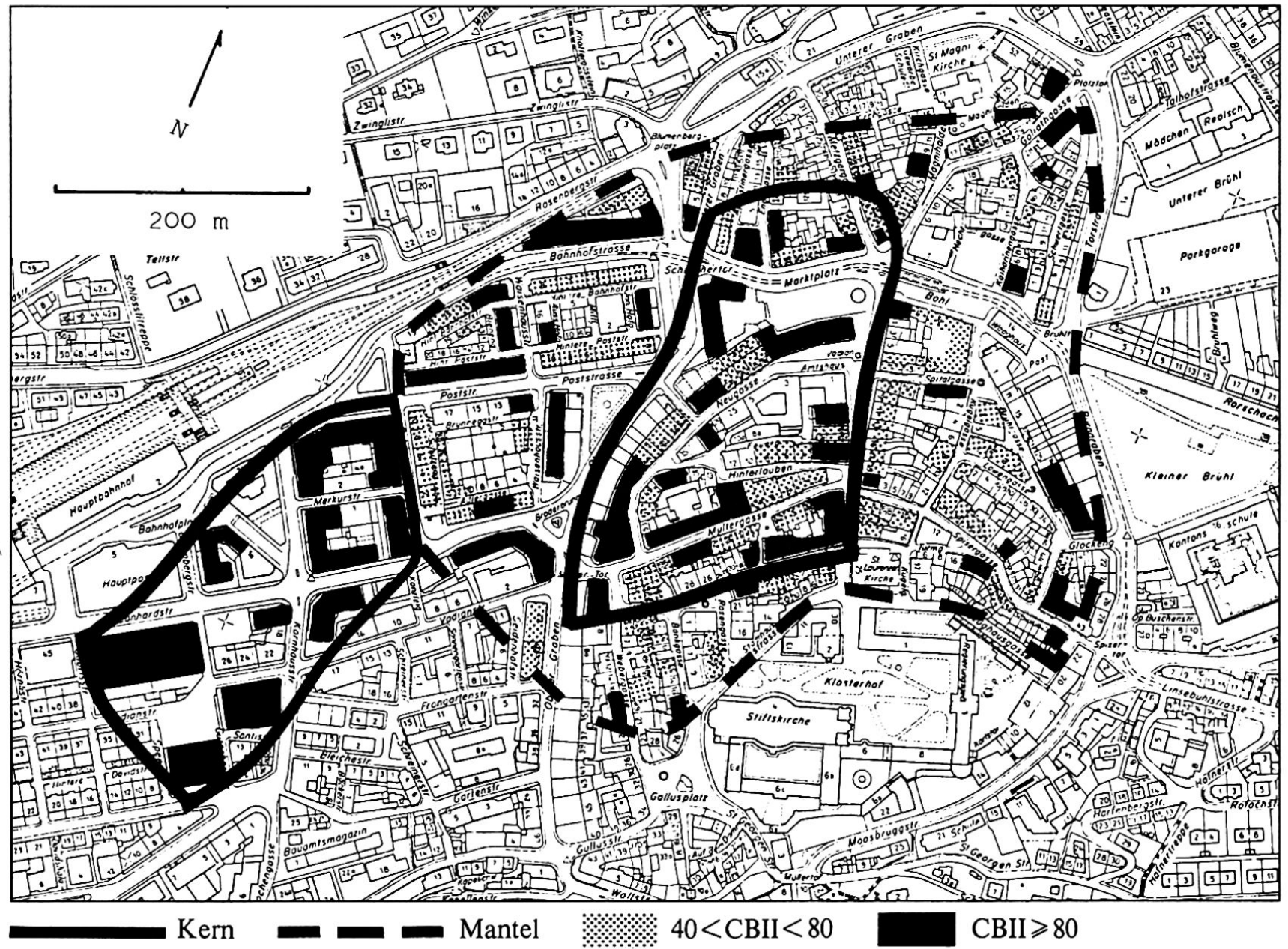

\section{Die innere Differenzierung der St. Galler Innenstadt}

Nach der Abgrenzung des St. Galler Geschäftszentrums haben wir untersucht, wie weit und in welchem Maße sich räumliche Ordnungsmuster ausgewählter Funktionsgruppen innerhalb des höchstwertigen Geschäftsgebietes erkennen lassen. In diesem Sinne wurden die Einzelhandelsfunktionen auf ihre räumliche Verteilung und Anordnung hin analysiert, weil sie «als die das Geschäftsgebiet ausmachenden Kernfunktionen angesehen werden können18).

Folgende Nutzungsgruppen wurden untersucht:

NG I Nahrungsmittel

NG II Kauf- und Warenhäuser
NG III a Spezialgeschäfte für Bekleidung (inkl. Textilgeschäfte)

NG III b Spezialgeschäfte für persönlichen Bedarf NG IV Spezialgeschäfte für Haushaltwaren, Wohnungseinrichtung und Freizeitbedarf NG V Dienstleistungsunternehmen des Einzelhandels.

Für jede einzelne dieser Nutzungsgruppen haben wir die sogenannte Intensivzone herausgearbeitet. Unter Intensivzone verstehen wir diejenigen Gebiete, in welchen die jeweils betrachtete Nutzungsgruppe überdurchschnittlich vertreten ist. Dabei wird als Kriterium nicht mehr (wie bei der Abgrenzung) die Geschoßflächennutzung verwendet, sondern die Länge der Schaufensterfronten. Diese Frontlängen wurden 
Abbildung 2: Intensivzone der Nutzungsgruppe IIIa im St. Galler Geschäftszentrum

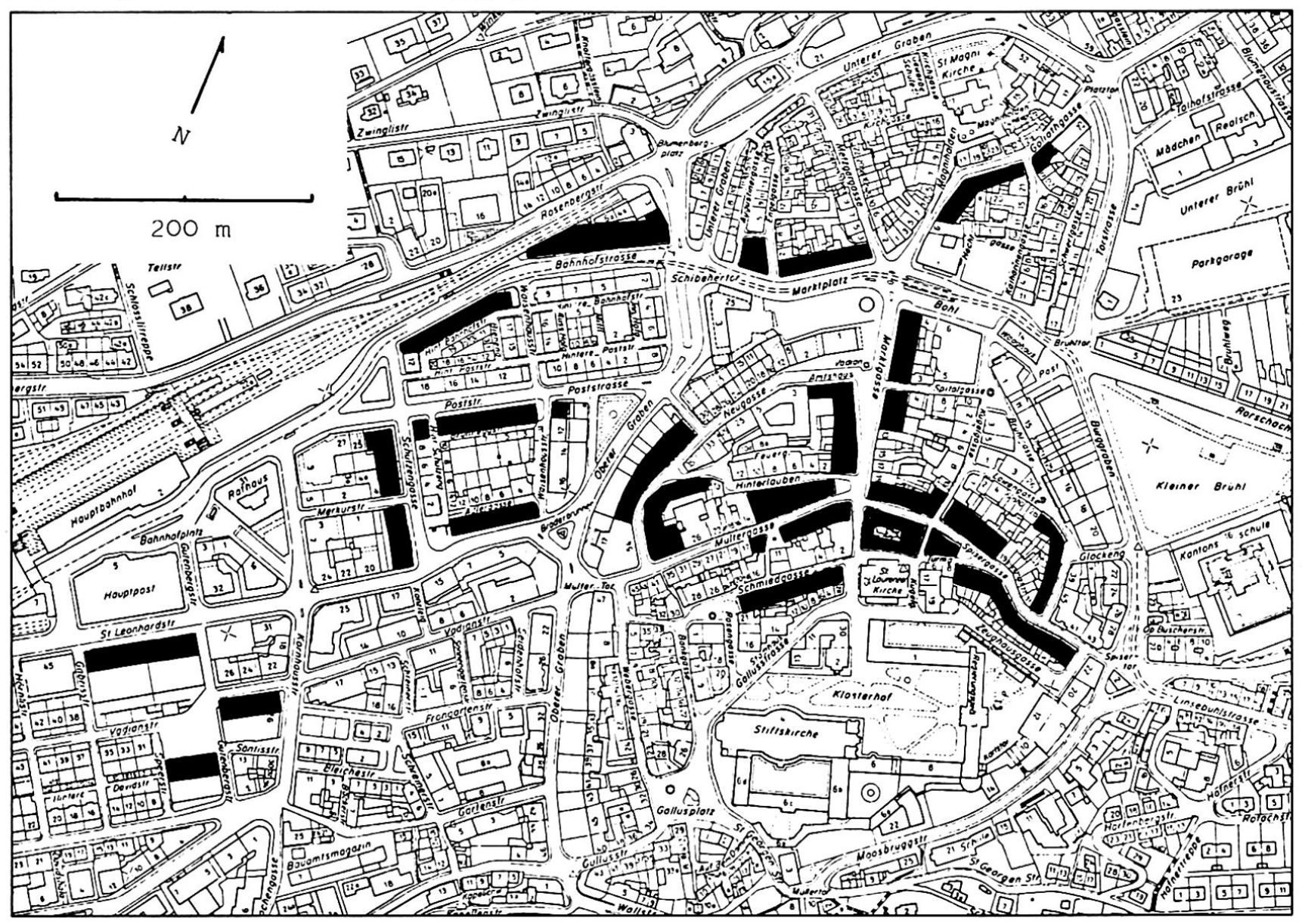

für alle insgesamt 81 Straßen-Abschnitte (à $100 \mathrm{~m}$ ) empirisch ermittelt; so beträgt beispielsweise die durchschnittliche Schaufensterfront in der NG III a (Spezialgeschäfte für Bekleidung) $22,8 \mathrm{~m}$ je $100 \mathrm{~m}$ Straße, in der Nutzungsgruppe I (Nahrungsmittel) hingegen lediglich 7,2 $\mathrm{m}^{19)}$.

Die aus diesen Analysen gewonnenen Erkenntnisse sind bemerkenswert: die Intensivzonen der NG II, IIIa und IIIb (d. h. Warenhäuser sowie hochspezialisierte Fachgeschäfte) fallen im südwestlichen Teil der Altstadt zusammen und bilden den Schwerpunkt der altstädtischen "City». Multergasse und Neugasse stellen den Kulminationspunkt dieser Einrichtungen in der Altstadt dar (vgl. Abb. 2).

Die Intensivzonen der NG I und IV schließen sich peripher an das höchstwertige Geschäftsgebiet an, sei es wegen geringerer Spezialisierung (Nahrungsmittel,
Grundversorgung für den lokalen Bedarf), sei es infolge größeren Raumbedarfs bei der NG IV.

Den Gegenpol zur Altstadt bildet das nach 1968 neu erstellte multifunktionale Zentrum Neumarkt im Bahnhofsviertel. Hier sind alle Nutzungen (mit Ausnahme der NG II) räumlich konzentriert. Im Gegensatz zur Altstadt fehlen aber jeweils mehrere Geschäfte mit ähnlichem Sortiment. Man muß deshalb hier von einem vollständigen Cityangebot ohne Konkurrenzverhältnis sprechen.

\section{Stadtgeographie - Stadtplanung}

Unter einem problemorientierten Blickwinkel soll Wissenschaft letztlich auch einen Beitrag leisten zur Lösung anstehender "Tagesprobleme». Die Skepsis gegenüber einer isolierten Wissenschaft, der Ruf nach 
gesellschaftlicher Relevanz hat nicht zuletzt auch bildungsökonomische Quellen; dabei darf aber der Nutzenbegriff nicht zu eng ausgelegt sein.

Der Erkenntniswert der Stadtgeographie, der Stadtforschung, liegt (neben der Herausarbeitung allgemeingültiger Konzepte) in der Bereitstellung von Entscheidungsgrundlagen für die Stadtplanung. Diese Aufgabe muß dabei keineswegs nur im Sinne einer operativen, direkt anwendungsorientierten Auftragsforschung verstanden werden; vielmehr sollten auch in unabhängigen Untersuchungen vermehrt normative Elemente (als solche deutlich erkennbar) enthalten sein. "Stadtplanung» ist dann nicht nur ein administrativer Vorgang, sondern ein allgemeiner Prozeß wachsenden Bewußtseins um städtische Probleme, Wertvorstellungen und Lösungsmöglichkeiten. Dabei kommt stadtgeographischen Arbeiten eine wichtige Katalysatorwirkung zu.

\section{Das Beispiel St. Gallen: Kommunikationsprobleme einer Innenstadt}

Im Sinne dieser Bemerkungen sollen im folgenden zwei Problemkreise näher beleuchtet werden, die beide mit der oben skizzierten funktionalen Bedeutung und Struktur der St. Galler Innenstadt eng verknüpft sind.

«Dienstleistungsfunktion», «Bedeutung als Zentraler Ort» kann ja nicht für sich allein stehen; damit unmittelbar verbunden sind Kommunikations- und Transportfunktionen, welche die Zentralität erst ermöglichen. Der Bedarf an Kommunikation (differenziert nach Volumen, Dynamik, Zeitverlauf und Aufteilung auf verschiedene Verkehrsträger) ist ein entscheidendes Element der Stadtplanung. Es ist offensichtlich, daß zuviel wie auch zuwenig Kommunikation die Zentrumsfunktion einer Innenstadt (zer)stören kann.

Das Wesentliche an einem zentralen Ort liegt ja wohl darin, daß auf einem Gang mehrere Dienstleistungsbedürfnisse erledigt werden können. Damit ist der Agglomerationseffekt eines Zentrums begründet. Das bedeutet, daß sich (ungeachtet der Transportart zum Zentrum hin) innerhalb desselben die Kommunikation auf der Nachfrageseite auf den Fußgängerverkehr abstützt.

In der St. Galler Innenstadt ergeben sich daraus zwei Probleme. Einmal bedeutet die oben dargelegte Zwei- kernigkeit mit einer ausgesprochenen Schwächezone zwischen den beiden Teilzentren, daß diese kaum als funktionale Einheit anzusprechen sind. Obwohl die Zwischenzone entlang St. Leonhard- und Vadianstraße kaum $300 \mathrm{~m}$ mißt, ist der Fußgängerstrom merklich schwächer als innerhalb der beiden Teilzentren. Die Massierung von Verwaltungsgebäuden des Bankund Versicherungssektors (mit einem vergleichsweise geringen Kundenvolumen) hat diese Entwicklung mitbestimmt. Die Frage ist offen, ob zwei Teilzentren gemeinsam nicht eine höhere Bedeutung erlangen könnten. Falls dies zutreffen sollte, müßte im Sinne der Zielsetzung «Stärkung der Stadt als Zentraler Ort»20) diese Schwächezone zwischen den beiden Kernen überwunden werden, und zwar sowohl durch die baulich-verkehrstechnische Gestaltung als Fußgängerzone wie auch durch die Förderung von Dienstleistungsangeboten.

Innerhalb der historischen Altstadt ergibt sich eine deutliche Dreiteilung, bedingt durch den privaten Fahrzeugverkehr auf Marktgasse und Marktplatz. Diese beiden Verkehrsachsen zerschneiden das an sich geschlossene Zentrum, was sich wiederum am Verhalten der Fußgänger bzw. am Volumen des Fußgängerstromes erkennen läßt. Konsequenterweise tritt eine deutliche Rangfolge der drei Teile zutage: das höchstwertige Zentrum ist das Gebiet um die Multergasse, gefolgt von der Spisergasse und schließlich der Iravorstadt nördlich des Marktplatzes. Dieses Bedeutungsgefälle könnte überwunden werden durch die Eliminierung der Trennungswirkung von Marktgasse und Marktplatz. Eine ganzheitliche Betrachtungsweise muß aber gleichzeitig zur Kenntnis nehmen, daß diesen Verkehrsströmen ebenfalls städtische Funktionen zukommen, die nicht einfach unterbunden werden können. Untersuchungen über die zweckmäßige Abstimmung von Fahrzeugverkehr und Fußgängerbedürfnissen sind für Entscheide, wie die Zerschneidung der Altstadt überwunden werden kann, unerläßlich.

\section{Literaturverzeichnis}

Akademie für Raumforschung und Landesplanung (Hrsg.): Handwörterbuch der Raumforschung und Raumordnung. Hannover, 1970.

BOESCH, M., SAUTER, J.: RP St. Gallen, Schlußbericht zur Übersicht. St. Gallen, 1972. 
CAROL, H.: The Hierarchy of Central Functions within the City. Annals, Ass. of Amer. Geogr. Vol. 50, 1960. CARTER, H.: The Study of Urban Geography. London, 1974.

DAVIES, D. H.: The Hard Core of Cape Town's CBD, An Attempt at Delimitation.

in: Economic Geography, Vol. 36, No. 1, 1960.

HOFMEISTER, B.: Stadtgeographie. Braunschweig, 1969.

JAEGER, F.: Strukturgutachten der Stadt St. Gallen, Bd. II/2. St. Gallen, 1975.

JETZER, J. P.: Verteilung und Hierarchie der zentralen Funktionen in der Stadt St. Gallen. Manuskript HSG, St. Gallen, 1975.

KANT, E.: Zur Frage der inneren Gliederung der Stadt. in: SCHÖLLER, P., Allgemeine Stadtgeographie. Wege der Forschung Band CLXXXI. Darmstadt, 1969.

LÖPFE, N.: St. Gallen morgen. Eine Einführung in den Generalverkehrsplan der Stadt St. Gallen. St. Gallen, 1967.

MURPHY, R. E., VANCE, J. E.: Delimiting the CBD. in: Economic Geography, Vol. 30, No. 3, 1954.

SOMBART, W.: Der moderne Kapitalismus. München/ Leipzig, 1916.

wOLF, K.: Geschäftszentren, Nutzung und Intensität als $\mathrm{Ma} ß$ städtischer Größenordnung. Ein empirischmethodischer Vergleich von 15 Städten der Bundesrepublik Deutschland. Rhein-Mainische Forschungen, Heft 72, Frankfurt a. M., 1971.

\section{Anmerkungen}

1) Praktikumsarbeiten und Exkursionsberichte, Manuskripte, Forschungsstelle für Wirtschaftsgeographie und Raumplanung der Hochschule St. Gallen, 1972 bis 1975.

2) JETZER, Zentrale Funktionen in der Stadt St. Gallen.

3) CAROL, Central Functions, p. 424.

4) HOFMEISTER, Stadtgeographie, S. 87.

5) KANT, Innere Gliederung der Stadt, S. 361.

7) Handwörterbuch der Raumforschung und Raumordnung, Sp. 149 ff.

8) SOMBART, Der moderne Kapitalismus, S. $131 \mathrm{f}$.

9) CARTER, Urban Geography, S. 54 f.

10) MURPHY/VANCE, Delimiting the CBD, S. $189 \mathrm{ff}$.
11) wOLF, Geschäftszentren, S. 32.

12) Von der Berechnung des bei MURPHY und VANCE verwendeten Höhenindexes (CBHI) konnte abgesehen werden, weil dieser bei ausgeglichener Geschoßzahl praktisch in einem konstanten Verhältnis zum CBII steht.

13) CARTER, Urban Geography, S. 203.

14) DAVIEs, Cape Town's CBD, S. 54 ff.

15) DAVIES wählte als Schwellenwerte $80 \% / 50 \%$.

16) LÖPFE, St. Gallen morgen, S. 22.

17) Lokale Verwaltung gilt bei DAVIES nicht als citybildend!

18) WOLF, Geschäftszentren, S. 25.

19) JETZER, Zentrale Funktionen in der Stadt St. Gallen, p. $26 \mathrm{ff}$.

20) BOESCH/SAUTER, RP St. Gallen, S. $38 \mathrm{ff}$. JAEGER, Strukturgutachten, S. $148 \mathrm{ff}$.

\section{Résumé: Le centre d'affaires de St-Gall}

St-Gall en tant que lieu central de rang «centre principal» comprend une zone d'influence d'environ 400000 habitants.

Différentes méthodes de délimitation et de structuration des centres ont été testées pour l'exemple de St-Gall par le groupe de recherche en géographie économique et aménagement du territoire de l'Ecole des Hautes Etudes Commerciales de St-Gall. Les deux illustrations en représentent les résultats: un index d'utilisation (central business intensity index) permet de délimiter deux sphères d'intensités différentes dans le centre d'affaires de St-Gall: soit un très intensif noyeau et un manteau un peu moins intensif. Une structuration du centre d'affaires résulte des zones intensives des différentes branches commerciales: il s'agit de sections du territoire considéré dans lesquelles ces branches sont numériquement particulièrement bien représentées.

L'analyse a montré une bipolarité du centre d'affaires: cet état de fait ainsi que ses conséquences pour la circulation piétonnière sont des facteurs très importants pour l'aménagement territorial de la ville. La circulation piétonnière se trouve en plusieurs endroits considérablement gênée: il s'avère nécessaire d'équilibrer les prétentions de cette dernière avec celles de la circulation des véhicules à moteur. 